\title{
Anti-osteoporosis Effect of 5-Bromo-2-(4-chlorobenzoyl)-(Z)-3-(2-cyano- 3-hydroxybut-2-enonyl)aminobenzo[b]furan: a Novel Selective Estrogen Receptor Modulator
}

\author{
Ryo Fukuyama ${ }^{1}$, Akio Shimokawa ${ }^{2}$, Yasushi Kodama ${ }^{3}$, Mitsugu Fujita ${ }^{1}$, Yoshitaka Ohishi ${ }^{4,5}$, Yuko Ando ${ }^{4}$, \\ Masao Koida ${ }^{6}$, and Hiromichi Nakamuta ${ }^{1, *}$ \\ ${ }^{1}$ Laboratory of Pharmacology, ${ }^{3}$ Laboratory of Molecular and Cellular Pharmacology, \\ Department of Pharmaceutical Sciences, Faculty of Pharmaceutical Sciences, Hiroshima International University, \\ 5-1-1 Hirokoshingai, Kure, Hiroshima 737-0112, Japan \\ ${ }^{2}$ Department of Pharmacy, National Hospital Organization Kanmon Medical Center, \\ 1-1 Chofusotoura-cho, Shimonoseki, Yamaguchi 752-8510, Japan \\ ${ }^{4}$ School of Pharmaceutical Sciences, Mukogawa Women's University, \\ Koshien Kyubancho, Nishinomiya, Hyogo 663-8179, Japan \\ ${ }^{5}$ School of Pharmaceutical Sciences, Doshisha Women's College of Liberal Arts, Kodo, Kyotanabe, Kyoto 610-0395, Japan \\ ${ }^{6}$ Department of Pharmacology, Faculty of Pharmaceutical Sciences, Setsunan University, \\ 45-1 Nagaotoge-cho, Hiraka, Osaka 573-0101, Japan
}

Received March 12, 2011; Accepted April 11, 2011

\begin{abstract}
The benzo[b]furan derivative MU314 inhibits in vitro bone resorption as potently as $\beta$-estradiol $\left(\mathrm{E}_{2}\right)$. Here, we examined the point of action on the anti-osteoporotic effects of MU314. MU314 (10 nM) suppressed lacunae formation by osteoclastic cells and ICI-182,780, a pure $\mathrm{E}_{2}$ antagonist, inhibited this effect. Specifically, we ovariectomized (OVX) Wistar female rats and subcutaneously injected them with either MU314 (30 or $100 \mu \mathrm{g} / \mathrm{kg})$ or $\mathrm{E}_{2}(100 \mu \mathrm{g} / \mathrm{kg})$ over an 8 -week period. Bone mineral content (BMC) in the proximal end of the tibia was significantly decreased (14\%) in OVX rats, and MU314 $(100 \mu \mathrm{g} / \mathrm{kg})$ and $\mathrm{E}_{2}$ significantly suppressed the decline in BMC. OVX rats exhibited decreased cancellous bone in the proximal end of the tibia and induced destruction of its trabecular structure. MU314 suppressed these changes. OVX also reduced the mechanical strength of the femoral neck, which was also recovered by MU314 and $E_{2} . E_{2}$ completely protected against OVX-induced uterine atrophy, but MU314 had no effect. These results strongly indicate that MU314 acts as a selective estrogen receptor modulator.
\end{abstract}

Keywords: selective estrogen receptor modulator, osteoporosis, osteoclast, ovariectomy, bone resorption

\section{Introduction}

Decreased circulating estrogen levels in postmenopausal women cause degenerative changes in the reproductive, skeletal, cardiovascular, and central nervous systems, among others $(1-3)$. In particular, the type and number of degenerative changes in the skeletal system leads to bone fragility, which can cause non-traumatic

*Corresponding author. h-nakamu@ps.hirokoku-u.ac.jp Published online in J-STAGE on May 21, 2011 (in advance) doi: 10.1254 /jphs.11049FP fractures in vertebra and the femoral neck $(4,5)$. Therefore, postmenopausal osteoporosis is a significant public health problem. In the United States of America, $\beta$-estradiol $\left(\mathrm{E}_{2}\right)$ has been employed for the treatment of postmenopausal syndromes in hormone replacement therapy (HRT). However, some meta-analyses have revealed a positive correlation between the duration of HRT and risk of breast cancer $(6,7)$.

This limitation of HRT prompted the development of a series of non-hormonal compounds that possess the beneficial effects of estrogen on skeletal tissue, but not its adverse effects on the reproductive organs such as 
breast and uterus. These types of compounds are called selective estrogen receptor modulators (SERMs). Raloxifene is a second-generation benzothiophene-derivative SERM and the sole compound approved for the prevention and treatment of postmenopausal osteoporosis $(8-10)$. Certain randomized clinical trials demonstrated that raloxifene decreased the fracture risk of vertebra in postmenopausal osteoporosis but led to side effects such as thromboembolic disorder $(11,12)$. These problems have in turn stimulated the development of next generation SERMs, which are more suitable for chronic therapy than raloxifene (9).

Recently, we synthesized a series of raloxifene derivatives in which the benzothiophene moiety was replaced by a benzofuran group; these derivatives were screened for bone resorption inhibition in in vitro assays. One such derivative, 5-bromo-2-(4-chlorobenzoyl)-(Z)-3-(2-cyano3-hydroxybut-2-enonyl)aminobenzo[b]furan, (Code No. MU314) was found to be as active as $E_{2}(13,14)$. In this study, we examined the mechanism of action of MU314 and its anti-osteoporosis activity to evaluate it as an SERM.

\section{Materials and Methods}

\section{Chemicals and laboratory animals}

The following reagents were obtained from the indicated sources: $\alpha$-MEM (Invitrogen, Auckland, New Zealand), FBS (Thermo Fisher Scientific, Waltham, MA, USA), receptor activator of nuclear factor- $\kappa \mathrm{B}$ ligand (RANKL) (Oriental Yeast Co., Ltd., Tokyo), M-CSF (R\&D Systems, Inc., Minneapolis, MN, USA), ICI182,780 (Tocris, Ellisville, MO, USA), and $\beta$-estradiol (Nacalai Tesque, Kyoto). Laboratory animals were purchased from Shizuoka Laboratory Animal Center (Shizuoka). MU314 was synthesized by Y. Ohishi, a co-worker in this study $(13,14)$.

\section{In vitro anti-bone resorption assay}

Monocyte-rich fractions were prepared from the tibiae and femora of ddY mice $(7-8$-weeks-old, $n=4)$. Bone marrow cells obtained from long bones were suspended in $\alpha$-MEM and layered onto Ficoll-Paque (GE Healthcare UK Ltd., Buckinghamshire, UK). After centrifugation, the fraction between the gel and supernatant was collected as the monocyte-rich fraction $\left(10^{5}\right.$ cells $)$ and cultured for $12 \mathrm{~h}$ in $\alpha$-MEM containing $10 \mathrm{ng} / \mathrm{ml} \mathrm{M-CSF}$ and $10 \%$ FBS. Subsequently, non-adherent cells $(2.5 \times$ $10^{5}$ cells/well) were inoculated into BioCoate Osteologic Discs (BD Biosciences, San Jose, CA, USA) in $\alpha$-MEM containing 10\% FBS, $20 \mathrm{ng} / \mathrm{ml} \mathrm{M-CSF}$, and $100 \mathrm{ng} / \mathrm{ml}$ RANKL and incubated for 7 days. $E_{2}$ and the other compounds were added to the medium at the beginning of culture. After cultivation, discs were rinsed with distilled water and cells dislodged with bleach solution ( 0.1 $\mathrm{M} \mathrm{NaOH}$ ). Resorption lacunae on the disc were evaluated by phase contrast microscopy and the areas of lacunae were measured as osteoclast resorption pits.

\section{Animal model for osteoporosis and drug treatment}

Forty Wistar female rats (12-weeks-old), which received ovariectomy (OVX) or sham operation under nembutal anesthesia, were divided into 5 groups and treated as described in Table $1 . \mathrm{E}_{2}$ and MU314 were dissolved and suspended in sesame oil (Wako Pure Chem., Inc., Osaka) and injected subcutaneously between 1 and 3 p.m., 4 days per week (Monday, Wednesday, Friday, and Saturday) over an 8-week period starting one week after surgery. All experiments involving animals were reviewed and approved by the Committee for Animal Experiments in Hiroshima International University.

Preparation of tissue samples and evaluation of antiosteoporosis

Rats were sacrificed by exsanguination through the femoral vein under nembutal anesthesia, and then the tibiae, femora, and uteri were surgically removed. Right tibiae were kept in $70 \%$ ice-cold ethanol solution before measuring bone mineral content (BMC) and bone mineral density (BMD). Left tibiae were embedded in watersoluble methacrylate resin (Wako Pure Chem.) to obtain $6-\mu \mathrm{m}$ longitudinal slices. Femora were evaluated for mechanical strength. Uteri (the proximal end of the vagina to the fallopian tubes) were washed in ice-cold saline and wet-tissue weights were measured. After fixation in $10 \%$ neutral formaldehyde, uteri were embedded in paraffin and sectioned for histological evaluation.

\section{$B M C$ and BMD measurements}

BMC and BMD were estimated by dual energy X-ray absorptiometry (DCS-600; Aloka, Osaka). The bone areas assessed were the entire and 5-mm-long proximal end of the right tibiae. Dual energy X-ray absorptiometry analyses (22 and $53 \mathrm{KeV}$ ) were conducted at $10 \mathrm{~mm} / \mathrm{s}$.

Table 1. Grouping and treatment

\begin{tabular}{lccl}
\hline Groups & N & Surgery & Treatment \\
\hline I & 8 & sham & vehicle \\
II & 8 & OVX & vehicle \\
III & 8 & OVX & $\mathrm{E}_{2}(100 \mu \mathrm{g} / \mathrm{kg}$, s.c. $)$ \\
IV & 8 & OVX & MU314 $(30 \mu \mathrm{g} / \mathrm{kg}$, s.c. $)$ \\
V & 8 & OVX & MU314 $(100 \mu \mathrm{g} / \mathrm{kg}$, s.c. $)$ \\
\hline
\end{tabular}


Assessing mechanical strength

The 28-mm long proximal end of the right femur was dissected and placed in a femoral neck holder (OsakaRika, Osaka) to fix the distal region by OSTORON II (GC Oc., Tokyo). The femur head was pressed to the breaking point (Max: $50 \mathrm{~kg}$ ) at a constant speed $(5 \mathrm{~mm} /$ $\mathrm{min})$, and the fracture load $(\mathrm{N})$ and fracture energy $(\mathrm{mJ})$ were calculated as indices of mechanical strength using Bone Breaking Strength Testers (KN-252C; Muromachi Kikai Co., Tokyo). The left femur was mounted on a 2-point-holder $(15 \mathrm{~mm})$ for the three-point bending test to measure the mechanical strength at the femoral center.

\section{Statistical analyses}

All the data were expressed as the mean and standard error (S.E.M.). Statistical analyses were performed by Fisher's least significant difference test. $P<0.05$ was defined as significant.

\section{Results}

The chemical structure of MU314 is shown in Fig. 1. This compound has a favorable balance of lipophilic and hydrophilic properties and benzo[b]furan derivatives with fused aromatic rings that exhibit certain unique<smiles>C/C(O)=C(\C#N)C(=O)Nc1c(C(=O)c2ccc(Cl)cc2)oc2ccc(Br)cc12</smiles>

Fig. 1. Chemical structure of MU314.
(A)

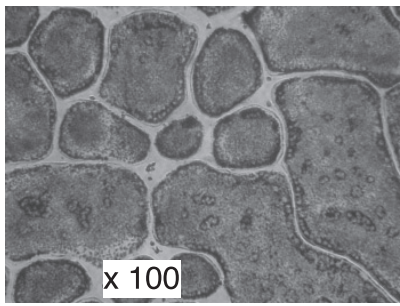

(B)
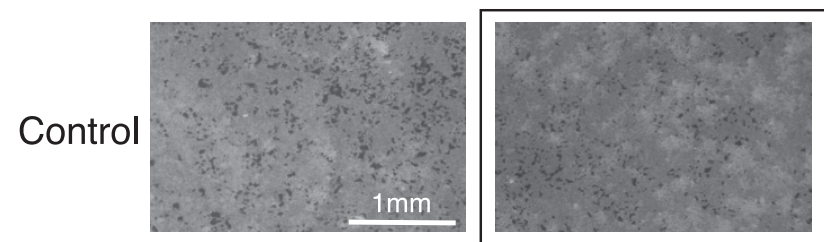

$\mathrm{E}_{2}$
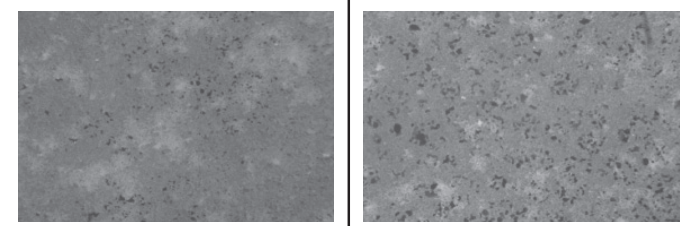

MU314
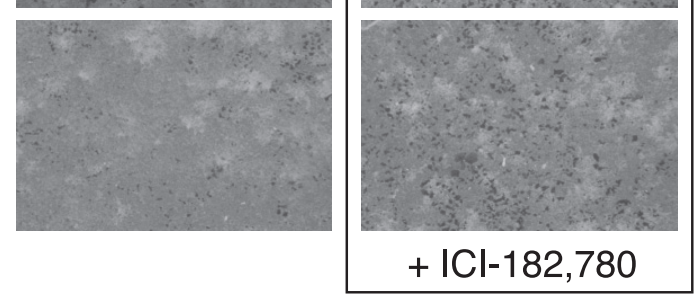

(C)

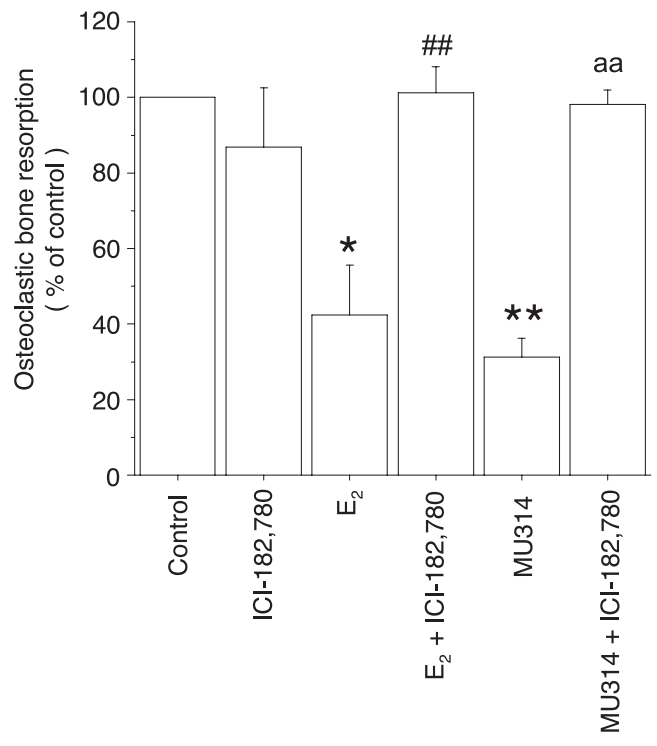

Fig. 2. Anti-bone resorption effects of MU314 in vitro. A) Osteoclast-like multinucleated giant cells were obtained from bone marrow cells cultured in the presence of M-CSF and RANKL. B) Phase contrast images of the surface of the Bio Coat Osteologic Disc. Dark spots visible on the surface of calcium phosphate thin films are the lacunae excavated by osteoclast-like multinucleated giant cells. C) Anti-bone resorption effect of MU314 (10 nM) and the effect of ICI-182,780 $(10 \mathrm{nM})$ on the anti-bone resorption effect of MU314. The area of the lacunae was calculated using image analysis software (Photoshop; Adobe) and osteoclastic bone resorption was estimated as a percentage of the Control. The average of the area of the lacunae on the plate and its standard error in the Control were 0.58 and $0.077 \mathrm{~mm}^{2}$, respectively $(\mathrm{n}=5) . * P<0.05$ and $* * P<0.01$ indicate significant differences against Controls. ${ }^{\#} P<0.01$, against the $\mathrm{E}_{2}$ group; ${ }^{\text {aa }} P<0.01$, against the MU314 group. 
bioactivities such as anti-human coronavirus activity and inhibition of ICAM-1 expression $(13,14)$.

By day 7, osteoclast-like multi-nuclear giant cells were observed in cultured bone marrow cells treated with RANKL and M-CSF (Fig. 2A) and number of lacunae, which were believed to be excavated by the osteoclastlike multi-nuclear giant cells, were seen on the surface of the mineralized calcium phosphate thin film coated on the culture vessel (Fig. 2B). $\mathrm{E}_{2}(10 \mathrm{nM})$ and MU314 (10 $\mathrm{nM}$ ) suppressed the formation of lacunae (Fig. 2C). ICI$182,780(10 \mathrm{nM})(15)$ inhibited the suppressive effect of MU314 on lacunae formation by the osteoclast-like multi-nuclear giant cells (Fig. 2C). ICI-182,780 exerted similar antagonistic effects on the suppressive effects of $\mathrm{E}_{2}$ (Fig. 2C).

Nine weeks post-OVX, the BMC of the proximal ends of tibiae were significantly diminished (14\%). $\mathrm{E}_{2}(100$ $\mu \mathrm{g} / \mathrm{kg}$, s.c.) prevented the OVX-induced decline of BMC
(A)

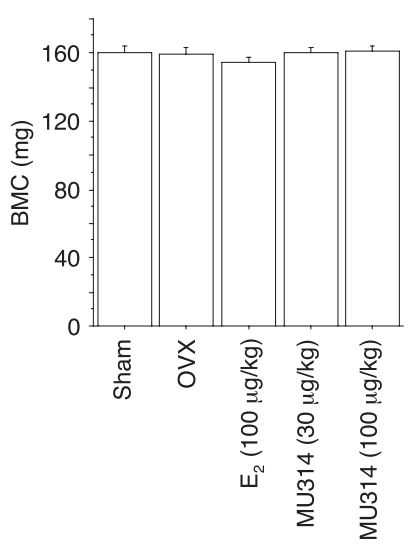

(C)

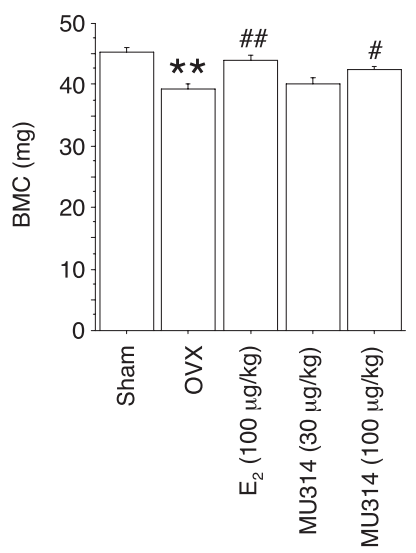

(B)

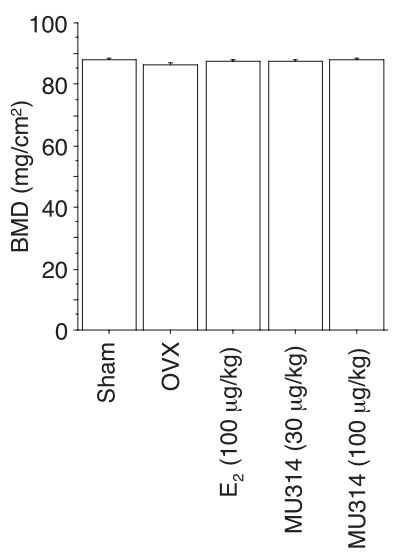

(D)

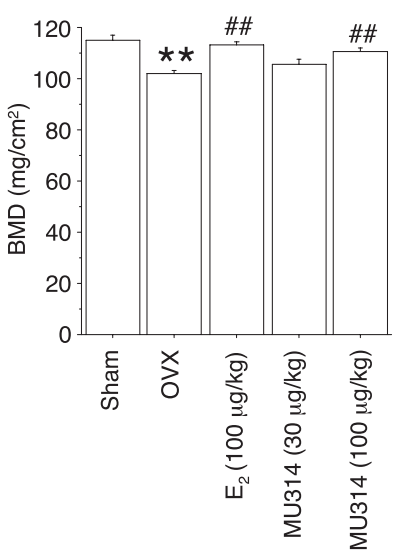

Fig. 3. Effect of MU314 on OVX-induced osteopenia. The upper two diagrams show the results for the entire tibia and the lower diagrams show the proximal end of the tibia. $* * P<0.01$ indicates significant difference against the Sham group. ${ }^{\#} P<0.05$ and ${ }^{\# \#} P<0.01$, against the OVX group.
(Fig. 3C). MU314 (100 $\mu \mathrm{g} / \mathrm{kg}$, s.c.) also significantly inhibited OVX-induced osteopenia (Fig. 3C). The changes in BMD correlated with those in BMC (Fig. 3D). The decrease in BMC and BMD by OVX was not detected in the entire tibia (Fig. 3: A and B). Observations of undecalcified bone sections indicated that cancellous bone volume was decreased and the trabecular structure of cancellous bone disappeared in the proximal tibiae of the metaphysis post-OVX (Fig. 4). $E_{2}$ and MU314 suppressed OVX-induced decreases in cancellous bone volume and the destruction of trabecular structure (Fig. 4).

OVX significantly decreased the fracture load of the femoral neck, and $\mathrm{E}_{2}$ completely prevented this decline (Fig. 5A). MU314, even at a low dose, protected against the decrease of mechanical strength in the femoral neck as potently as $\mathrm{E}_{2}$ (Fig. $5 \mathrm{~A}$ ). The change in fracture energy of the femoral neck was similar to that of the fracture load, but no significant change was observed (Fig. 5B) between the sham and OVX groups. The three-point
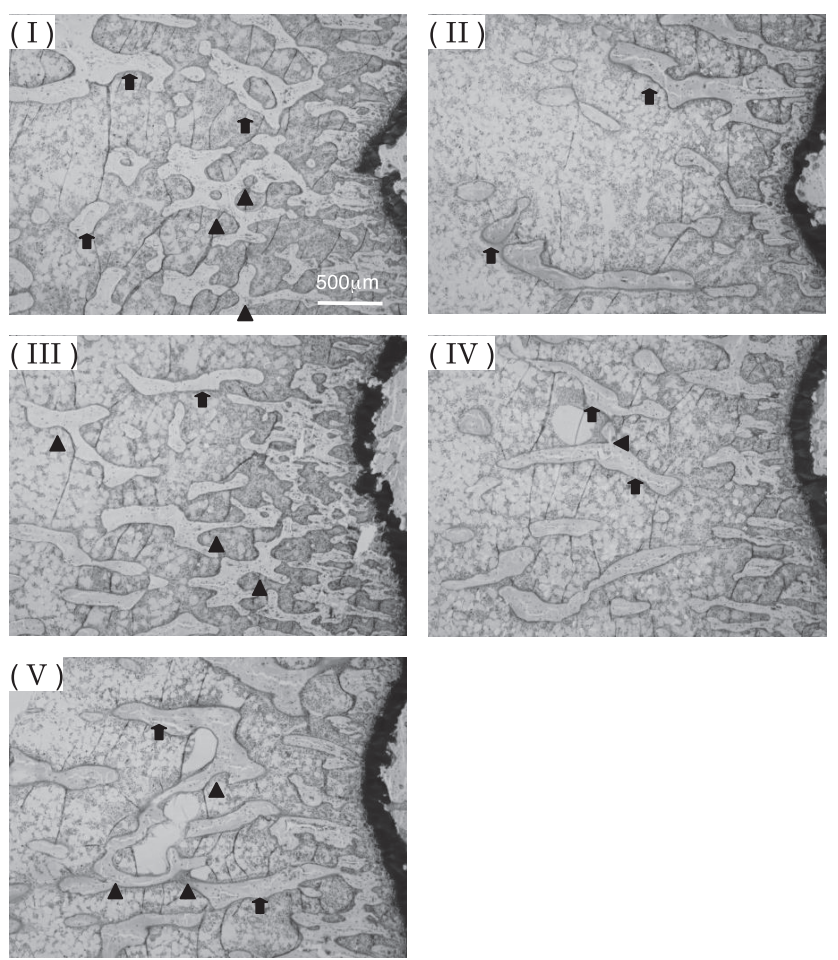

Fig. 4. Images of cancellous bone on the proximal tibia metaphysis in an undecalcified tissue section. Cancellous bone tightly framed the trabecular structure in the Sham group. The reduction in cancellous bone by OVX induced the loss of connectivity between cancellous bone and the destruction of trabecular structure. I: Sham group, II: OVX group, III: OVX + $\mathrm{E}_{2}(100 \mu \mathrm{g} / \mathrm{kg}$, s.c. $)$ group, IV: OVX + MU314 (30 $\mu \mathrm{g} / \mathrm{kg}$, s.c.) group, and V: OVX $+\mathrm{MU} 314(100 \mu \mathrm{g} / \mathrm{kg}$, s.c.) group. The arrow and arrowhead indicate cancellous bone and the connectivity between cancellous bones, respectively. 
bending test of the femoral shaft failed to detect OVXinduced loss of mechanical strength (Fig. 5: C and D).

Because of $\mathrm{E}_{2}$ administration, the OVX-induced decrease in uterine weight was completely prevented (Fig. 6A), whereas MU314 had no effect on OVX-induced uterine atrophy (Fig. 6A). Histological examination of paraffin sections showed the endometrial dystrophy caused by OVX, whereas $\mathrm{E}_{2}$ had a potent hypertrophic effect (Fig. 6B). In contrast, MU314 did not have an $\mathrm{E}_{2}$ like stimulating effect on the endometrium (Fig. 6B).

\section{Discussion}

Osteoclast progenitor cells in the bone marrow differentiate and mature into osteoclasts in the presence of RANKL and M-CSF in vitro $(16,17)$. Osteoclast-like multi-nuclear giant cells appeared after 3 days of incuba-
(A)

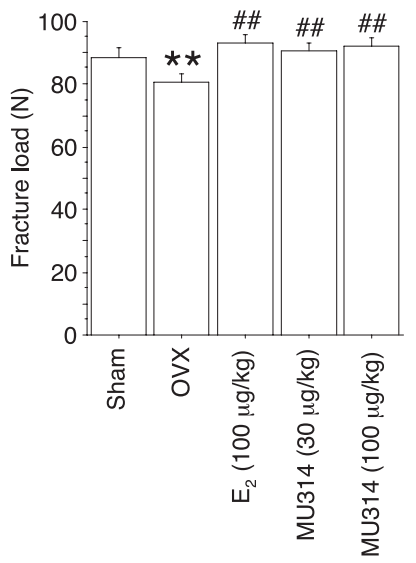

(C)

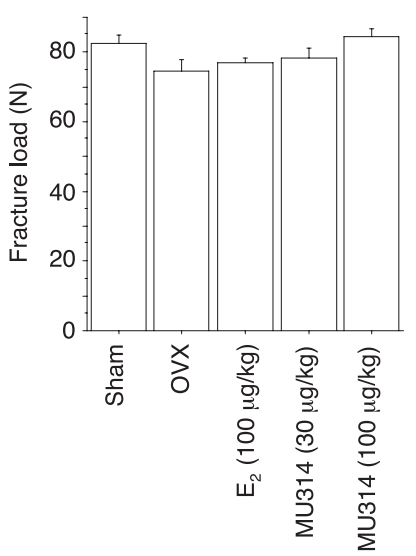

(B)

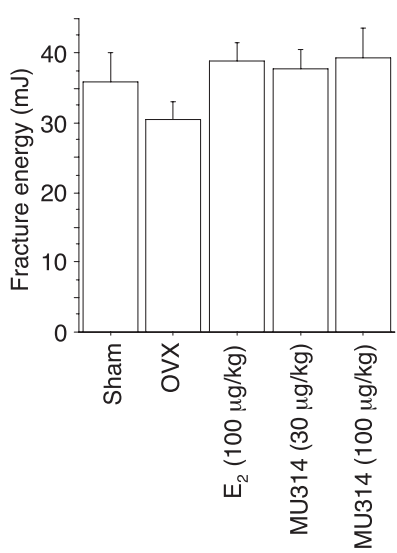

(D)

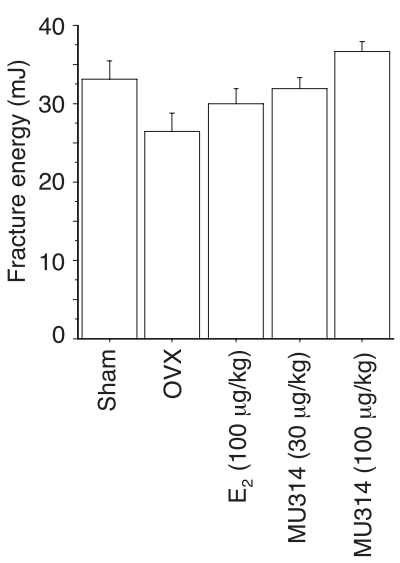

Fig. 5. Effects of MU314 on OVX-induced decrease in mechanical strength. The upper diagrams show the mechanical strengths of femoral necks (A, B) and the lower diagrams show those of the femur shaft (C, D). $* * P<0.01$ indicates a significant difference against the Sham group; ${ }^{\#} P<0.01$ against the OVX group. tion and expressed the tartrate-resistant acid phosphatase (data not shown), an osteoclast biomarker (18). Several lacunae appeared on the surface of the mineralized calcium phosphate thin-film after 7 days of incubation (Fig. 2B). Therefore, we conclude that osteoclast-like multinuclear giant cells in this culture system mediated bone
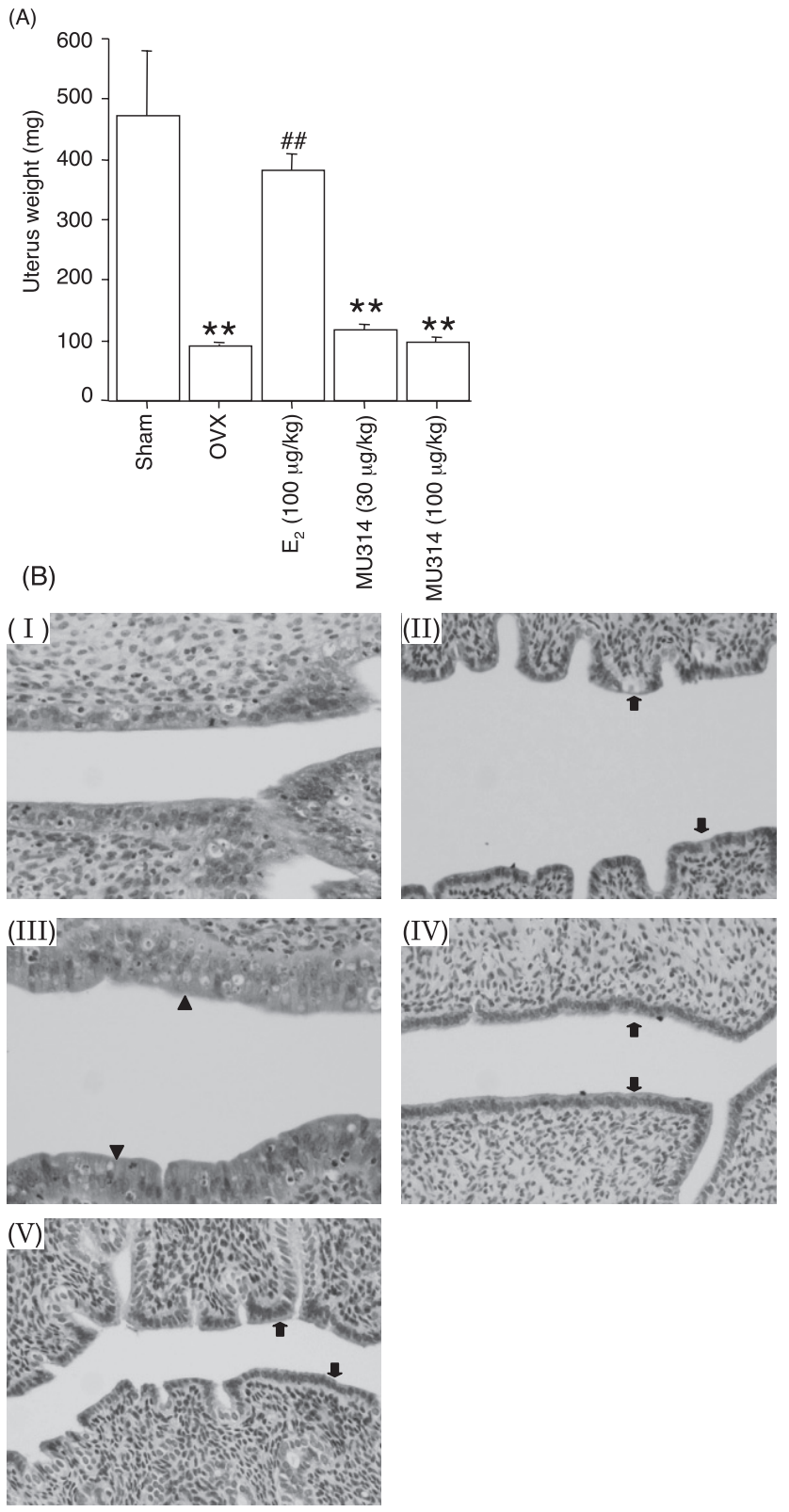

Fig. 6. Effect of MU314 on OVX-induced uterine atrophy. A) The weight of the uterus at 9 weeks after surgery. $* * P<0.05$ indicates a significant difference against the Sham group. ${ }^{\#} P<0.01$, against the OVX group. B) Histological images of endometrium. I: Sham, II: OVX, III: OVX + E $2(100 \mu \mathrm{g} / \mathrm{kg}$, s.c. $)$ group, IV: OVX + MU314 (30 $\mu \mathrm{g} / \mathrm{kg}$, s.c.) group, and V: OVX + MU314 (100 $\mu \mathrm{g} / \mathrm{kg}$, s.c.) group. Arrows indicate the region of endometrial atrophy and arrow heads indicate the region of endometrial hypertrophy. 
resorption.

MU314 inhibited bone resorption as potently as $E_{2}$. The inhibitory effect of MU314 on lacunae formation by osteoclasts was attenuated by ICI-182,780, a pure $\mathrm{E}_{2}$ antagonist (15). Similarly, ICI-182,780 suppressed the inhibitory effects of $E_{2}$. Therefore, these results strongly indicate that MU314 exerts anti-bone resorption activity by stimulating the estrogen receptor (ER).

OVX-rats exhibit similar pathophysiological changes in bone metabolism as postmenopausal women and therefore are frequently used in drug development studies as a model for postmenopausal osteoporosis $(19,20)$. Evaluation of tibial bone mass by dual energy X-ray absorptiometry showed that the bone loss in OVX-rat occurred mainly at the proximal end. Histological observation of undecalcified thin sections revealed that the decreased bone mass resulted from the decline of cancellous bone volume and disappearance of trabecular structure in the proximal tibia of the metaphysis. These changes in bone volume and trabecular structure were completely inhibited by MU314 or $\mathrm{E}_{2}$.

Clinical studies indicate that the degenerative changes of cancellous bone in quantity and quality occur in the neck of femora and lumbar vertebra and cause nontraumatic fracture in postmenopausal women $(21,22)$. Assessments of mechanical strength showed that OVX decreased the fracture load of the femoral neck and that MU314 and $E_{2}$ suppressed the OVX-induced decline in bone mechanical strength. A similar reduction on fracture load was not detected in the femoral shaft, which did not show a decrease in bone mass. Fracture load is one of the main indices for assessing mechanical strength (23). Therefore, MU314 may also suppress OVX-induced decrease in the mechanical strength of the bone.

$\mathrm{E}_{2}$ stimulates reproductive tissues and increases the risk of uterine and breast cancers in postmenopausal women (7). In this experiment, $\mathrm{E}_{2}$ had a potent hypertrophic effect on the endometrium. In contrast, MU314 did not stimulate OVX-induced uterine atrophy. These results strongly indicate that MU314 has SERM-like characteristics.

The osteoprotective effects of $E_{2}$ are well known, but its mechanism of action has not been completely elucidated. Nakamura et al. demonstrated that $E_{2}$ activates ER- $\alpha$ in mature osteoclasts and induces their apoptosis by stimulating the Fas/FasL system in a gene-disruption model in which ER- $\alpha$ ablation was specifically targeted to the differentiated osteoclasts (24). Therefore, they concluded that OVX-induced rapid enhancement of osteoclastic bone resorption could be explained by the increase of osteoclast survival because of $E_{2}$ deficiency. In contrast, Robinson et al. showed that $\mathrm{E}_{2}$ exerts osteoprotective effects by inhibiting RANKL-stimulated osteo- clastic differentiation (25). In a preliminary study using an osteoporosis mouse model, we showed that OVX decreased FasL expression in bone tissue, and $E_{2}$ or MU314 suppressed the reduction of FasL expression by OVX (data not shown). We therefore consider that the anti-bone resorption activity of MU314 occurs by decreasing the life span of osteoclasts by a mechanism that would include apoptotic osteoclast death.

Raloxifene is the sole SERM that reduces vertebral fracture risk of postmenopausal women. However, there is growing evidence that raloxifene treatment has no effect on the fracture risk of nonvertebral regions and increases adverse effects such as thromboembolic disorder $(11,12)$. These problems promote the development of a new generation SERMs that have beneficial properties for the therapy of osteoporosis.

In conclusion, MU314 is a novel compound that exhibits SERM-like characteristics and may become a principal compound in the development of anti-osteoporotic therapies.

\section{Acknowledgments}

We thank Mr. Koichiro Saisho, Mr. Yosuke Ikeda, and Miss Chihiro Kobayashi for their valuable assistance.

\section{References}

1 Loose DS, Stancel GM. Estrogens and progestins. In: Brunton LL, Lazo JS, Parker KL, editors. Goodman \& Gilman's the pharmacological basis of therapeutic. 8th ed. New York: McgrawHill; 2006. p. 1541-1572.

2 Rexrode KM. Emerging risk factors in women. Stroke. 2010; 41:S9-S11.

3 Schmidt PJ, Rubinow DR. Sex hormones and mood in the perimenopause. Ann N Y Acad Sci. 2009;1179:70-85.

4 Lindsay R, Cosman F. Osteoporosis. In: Braunwald E, Fauci AS, Kasper DL, Hauser SL, Longo DL, Jameson JL, editors. Harrison's principles of internal medicine. 15th ed. New York: McGraw-Hill; 2001. p. 2226-2237.

5 Sambrook P, Cooper C. Osteoporosis. Lancet. 2006;367:2010 2018.

6 Colditz GA, Egan KM, Stampfer MJ. Hormone replacement therapy and risk of breast cancer: results from epidemiologic studies. Am J Obstet Gynecol. 1993;168:1473-1480.

7 Colditz GA, Hankinson SE, Hunter DJ, Willett WG, Manson JE, Stampeer MJ, et al. The use of estrogens and progestins and the risk of breast cancer in postmenopausal women. N Engl J Med. 1995;332:1589-1593.

8 Grese TA, Sluka JP, Bryant HU, Culliman GL, Glasebrook AL, Jones $\mathrm{CD}$, et al. Molecular determinants of tissue selectivity in estrogen receptor modulators. Proc Natl Acad Sci U S A. 1997; 94:14105-14110.

9 Taylor HS. Designing the ideal selective estrogen receptor modulator - an achievable goal? Menopause. 2009;16:609-615.

10 Suzuki A, Sekiguchi A, Asano S, Itoh M. Pharmacological topics of bone metabolism: recent advances in pharmacological man- 
agement of osteoporosis. J Pharmacol Sci. 2008;106:530-535.

11 Ettinger B, Black DM, Mitlak BH, Knickerbocker RK, Nickelsen $\mathrm{T}$, Genant HK, et al. Reduction of vertebral fracture risk in postmenopausal women with osteoporosis treated with raloxifene: results from a 3-year randomized clinical trial. JAMA. 1999; 282:637-645.

12 Cranney A, Adachi JD. Benefit-risk assessment of raloxifene in postmenopausal osteoporosis. Drug Sat. 2005;28:721-730.

13 Ando Y, Ando K, Yamaguchi M, Kunitomo J, Koida M, Fukuyama R, et al. A novel oxazine ring closure reaction affording (Z-((E)-2-styrylbenzo[b]furo[3,2-d][1,3] oxazin-4-ylideno) acetaldehydes and their anti-osteoclastic bone resorption activity. Bioorg Med Chem Lett. 2006;16:5849-5854.

14 Tabuchi Y, Ando Y, Kanemura I, Ohishi T, Koida M, Fukuyama $\mathrm{R}$, et al. Preparation of novel (Z)-4-ylidenebenzo[b]furo[3,2-d] $[1,3]$ oxazines and their biological activity. Bioorg Med Chem. 2009;17:3959-3967.

15 Wakeling AE, Bowler J. ICI 182,780, a new antioestrogen with clinical potential. J Steroid Biochem Mol Biol. 1992;43:173177.

16 Anderson DM, Maraskovsky E, Billingsley WL, Dougall WC, Tometsko ME, Roux ER, et al. A homologue of the TNF receptor and its ligand enhance T-cell growth and dendritic-cell function. Nature. 1997;390:175-179.

17 Yasuda H, Shima N, Nakagawa N, Yamaguchi K, Kinosaki M, Mochizuki S, et al. Osteoclast differentiation factor is a ligand for osteoprotegerin/osteoclastogenesis-inhibitory factor and its identical to TRANCE/RANKL. Proc Natl Acad Sci U S A. 1998;
95:3597-3602.

18 Udagawa N, Takahashi N, Akatsu T, Tanaka H, Sasaki T, Nishihara T, et al. Origin of osteoclasts: Mature monocytes and macrophage are capable of differentiating into osteoclasts under a suitable microenvironment prepared by bone marrow-derived stromal cells. Proc Natl Acad Sci U S A. 1990;87:7260-7264.

19 Kalu DN. The ovariectomized rat model of postmenopausal bone loss. Bone Miner. 1991;15:175-191.

20 Nitta T, Fukushima T, Nakamuta H, Koida M. Glucocorticoidinduced secondary osteopenia in female rats: A time course study as compared with ovariectomy-induced osteopenia and response to salmon calicitonin. Jpn J Pharmacol. 1999;79:379-386.

21 Atkinson PJ. Variation in trabecular structure of vertebrae with age. Calcif Tissue Res. 1967;1:24-32.

22 Rodin A, Murby B, Smith MA, Caleffi M, Fentiman I, Chapman MG, et al. Premenopausal bone loss in the lumbar spine and neck of femur: a study of 225 Caucasian women. Bone. 1990;11:1-5.

23 Turner $\mathrm{CH}$, Burr DB. Basic biomechanical measurements of bone: A tutorial. Bone. 1993;14:595-608.

24 Nakamura T, Imai Y, Matsumoto T, Sato S, Takeuchi K, Igarashi $\mathrm{K}$, et al. Estrogen prevents bone loss via estrogen receptor a and induction of Fas ligand in osteoclasts. Cell. 2007;130:811-823.

25 Robinson LJ, Yaroslavskiy BB, Griswold RD, Zadorozny EV, Guo 1, Tourkova IL, et al. Estrogen inhibits RANKL-stimulated osteoclastic differentiation of human monocytes through estrogen and RANKL-regulated interaction of estrogen receptor-alpha with BCAR1 and Traf6. Exp Cell Res. 2009;315:1287-1301. 